\title{
Competencias Digitales de profesionales que inician su carrera universitaria como docentes.
}

\author{
Erika Machado \\ profesoraerika@gmail.com \\ http://orcid.org/0000-0002-5277-6543 \\ Sistema de Actualización Docente del Profesorado (SADPRO-UCV) \\ Universidad Central de Venezuela \\ Caracas, Venezuela.
}

Recibido: 11/08/2020 Aceptado: 12/02/2021

\begin{abstract}
Resumen
Este estudio trata sobre el uso de las competencias digitales por profesionales que inician su carrera como docentes universitarios, cursantes del Diplomado de Formación Integral para el Docente de la Universidad Central de Venezuela (Aletheia). Es una investigación cuantitativa, descriptiva, participaron 89 profesores de la novena (2018-2019) y décima cohortes (20192020) del diplomado. Los datos fueron recogidos a través de un cuestionario en línea. Su análisis mostró que estos profesionales usan en un nivel básico e intermedio las competencias digitales distribuidas en las áreas de Información y alfabetización informacional, Comunicación y colaboración, Creación de contenido digital, Seguridad y Resolución de problemas de las competencias digitales. Utilizan el correo electrónico como una herramienta frecuente para los procesos de comunicación y manejan las herramientas tecnológicas disponibles en la universidad (Campus Virtual, Biblioteca Virtual y Repositorio Institucional Saber UCV). Con estos resultados se podría sustentar el diseño de un plan de formación y capacitación dirigido a mejorar, actualizar y desarrollar competencias tecnológicas que faciliten la labor docente, optimicen los recursos de la universidad y beneficien a la población estudiantil.
\end{abstract}

Palabras clave: competencias digitales, docentes universitarios, tecnologías de la información y comunicación, capacitación, formación docente, praxis docente.

\section{Competências digitais de profissionais que iniciam a carreira universitária como professores.}

\section{Resumo}

Este estudo trata do uso de competências digitais por profissionais que iniciam suas carreiras como professores universitários, alunos do Diploma de Formação Integral de Professores da Universidade Central da Venezuela (Aletheia). Trata-se de uma pesquisa quantitativa, descritiva, com participação de 89 docentes da nona (2018-2019) e décima coortes (2019-2020) do diploma. Os dados foram coletados por meio de questionário online. A sua análise revelou que estes profissionais utilizam competências digitais distribuídas a um nível básico e intermédio nas áreas da informação e literacia informacional, Comunicação e colaboração, Criação de conteúdos digitais, Segurança e Resolução de problemas de competências digitais. Utilizam o email como ferramenta frequente para os processos de comunicação e tratam das ferramentas tecnológicas disponíveis na universidade (Campus Virtual, Biblioteca Virtual e 
Repositório Institucional Saber UCV). Com estes resultados, poderá ser apoiada a concepção de um plano de formação e formação visando a melhoria, atualização e desenvolvimento de competências tecnológicas que facilitem o ensino, otimizem os recursos universitários e beneficiem a população estudantil.

Palavras-chave: habilidades digitais, professores universitários, tecnologias da informação e comunicação, formação, formação de professores, prática docente.

\title{
Digital Competences of professionals who start their university careers as teachers.
}

\begin{abstract}
This study deals with the use of digital skills by professionals who start their careers as university teachers, students of the Comprehensive Teacher Training Diploma at the Central University of Venezuela (Aletheia). It is a quantitative, descriptive research, 89 professors of the ninth (2018-2019) and tenth cohorts (2019-2020) of the diploma participated. The data was collected through an online questionnaire. Their analysis showed that these professionals use at a basic and intermediate level the digital competences distributed in the areas of Information and information literacy, Communication and collaboration, Creation of digital content, Security and Problem solving of digital competences. They use email as a frequent tool for communication processes and handle the technological tools available at the university (Virtual Campus, Virtual Library and Institutional Repository Saber UCV). With these results, the design of a training and training plan aimed at improving, updating and developing technological skills that facilitate teaching work, optimize university resources and benefit the student population, could be supported.
\end{abstract}

Keywords: digital skills, university teachers, information and communication technologies, training, teacher training, teaching practice ...

\section{Introducción.}

Ante la creciente demanda del uso de las Tecnologías de la Información y Comunicación (TIC) en los diferentes entornos del ser humano, la enseñanza y el aprendizaje no pueden quedar fuera del desarrollo y proceso de transformación tecnológica, que vive la sociedad actual. El uso adecuado, eficiente y eficaz de las tecnologías, hace a las personas aptas, para un mundo tan cambiante y acelerado como las tecnologías mismas. De allí pues, que la educación, debe tener como fin formar ciudadanos preparados para ver y trabajar en la denominada sociedad de la información (Levis, 2006).

Ante esta situación, la educación debe adecuarse a las necesidades de la sociedad de la información, de los nativos digitales, quienes necesitan utilizar la tecnología en su formación académica. De allí la importancia de las TIC para la labor profesional, de esta manera, se 
estimula que el docente de educación secundaria pueda transformar e innovar los procesos de enseñanza y de aprendizaje a los que se enfrenta en su quehacer cotidiano (Larrea, 2013).

Los docentes requieren de un proceso de formación y actualización, que les permita, no solo adquirir las competencias digitales, sino comprenderlas, transformarlas, adaptarlas/adecuarlas y utilizarlas, como una herramienta y recurso que favorezca los procesos de enseñanza y de aprendizaje en su praxis profesional.

De ahí que debemos destacar la importancia de la formación previa y continua de los docentes, en lo que respecta al uso de las tecnologías, puesto que la calidad educativa en un país tiene su origen en la calidad de la formación y aprendizaje permanente de sus profesores. Si son ellos los encargados de los procesos de formación de los jóvenes en el uso y manejo adecuado de las TIC, entonces, es prioritario pensar en la necesidad de formar a los educadores en este aspecto. Podemos decir que "Los profesores no aplican los métodos que les son predicados sino que aplican los métodos que le son aplicados” (Fernández, 2010, p. 20).

Ahora bien, la praxis docente, según Castro y otros (2006) se puede entender como:

\begin{abstract}
Una acción que permite innovar, profundizar y transformar el proceso de enseñanza del docente en el aula. La práctica docente está unida a la realidad del aula, debido a que todo lo que hace el docente se refiere a lo que se hace en la vida cotidiana en la escuela, esta inscripción hace posible una producción de conocimientos a partir del abordaje de la práctica docente como un objeto de conocimiento, para los sujetos que intervienen, por eso la práctica se debe delimitar en el orden de la praxis como proceso de comprensión, creación y transformación de un aspecto de la realidad educativa. (p.24).
\end{abstract}

Tomando esta definición en consideración, se puede decir que la praxis docente comprende una serie de decisiones aplicables al trabajo cotidiano, dentro de los procesos de enseñanza y de aprendizaje; que debería ser innovadora, transformadora y capaz de adaptarse tecnológicamente al ámbito social.

De allí, la necesidad de conocer el uso y conocimiento de las competencias digitales en actividades educativas, ya que, como entes conductores del proceso, son responsables de incorporar las tecnologías al proceso de enseñanza y aprendizaje con fines pedagógicos. Esta idea conduce a la necesidad de la formación tecnológica con fines pedagógicos; donde el profesional que se incorpora a la docencia universitaria, desarrolle un conjunto de habilidades, destrezas y competencias tecnológicas, que lo lleven a afrontar con eficacia y eficiencia, las nuevas circunstancias educativas. 


\section{Marco Referencial}

En la sociedad actual del siglo XXI, los seres humanos viven sumergidos en una comunidad globalizada con altos niveles de tecnificación e innovación, que tienen incidencia en diversos espacios y ámbitos del quehacer social, donde los individuos pasan a ser partícipes de lo que se ha denominado Sociedad de la Información, que en conjunto con las Tecnologías de la Información y Comunicación (TIC), transforman el desempeño de las personas en sus actividades cotidianas, y en particular el ámbito educativo no escapa de una realidad tan avasallante (UNESCO, 2008).

Es debido a esto que se ha venido observando que las TIC han tomado un papel con tanta relevancia e importancia en los procesos educativos (Pere Márques, 2000). Han resultado ser un pilar dentro de la acción de formativa de los estudiantes, para lo cual se emplean un conjunto de herramientas tecnológicas, que sirven de apoyo y canal para facilitar los procesos de enseñanza y aprendizaje. En estos momentos se consideran elementos prioritarios para acceder a diversidad de información y a través de la comunicación se comparte sin tantas limitaciones de tiempo y espacio (Bosco, 2013).

De allí pues, que uno de los principales beneficios y ventajas de la tecnología, es que se han constituido en un canal de comunicación inmediata, que reduce considerablemente las distancias y permite además aprovechar el tiempo, llegando a facilitar la relación entre estudiante y profesor, sin embargo, los múltiples beneficios que ofrecen las tecnologías, deberían ir a la par con la formación del profesorado, para que las TIC se transformen en un verdadero canal de comunicación inmediato, lo cual implica que el profesor modifique y cambie su visión y manera de trabajar con tecnología.

Acaso (2013) señaló:

Mientras todo cambia y especialmente los sectores e industrias relacionados con la gestión del conocimiento, el mundo de la educación permanece igual que hace mucho tiempo, anclado en un paradigma más cercano al siglo XIX y a la producción industrial que las dinámicas propias del siglo XXI... (p.28)

Cabe destacar la importancia que tiene la formación previa, constante y actualizada de los docentes, sobre todo en lo referido al conocimiento, uso e implementación de las tecnologías en el proceso de instrucción, pues mucho de la calidad y alto nivel educativo de un país, tiene su inicio y origen en la calidad de la formación del docente (Badillo, 2016). 
Se puede decir que "los profesores no aplican los métodos que le son predicados, sino que aplican los métodos que le son aplicados" (Fernández, 2010, p.20). Esto nos dice, que si queremos docentes que formen en el conocimiento y uso de la tecnología, los primeros en poseer el conocimiento, habilidades, destrezas y competencias tecnológicas, deben ser ellos. Prendes (2001) señala que:

En medio de todo este mundo cambiante nos encontramos con un sistema escolar que está poniendo a los maestros en el centro de la encrucijada, pues son considerados como una de las claves del cambio. Cambio que además de necesario se está contemplando como urgente, pues nos encontramos actualmente con estructuras y modelos obsoletos que no parecen responder ni a los alumnos ni a la sociedad ni a las demandas del mercado de trabajo. (p.1)

Por otra parte, Martínez, Leite y Monteiro (2000) señalan que "la formación inicial de profesores de enseñanza básica es un tema al que se presta mucha atención pues de ella depende la calidad de los aprendizajes de las futuras generaciones". (p.70)

Se puede decir que el trabajo docente involucra un conjunto de decisiones, aplicables a la labor diaria, dentro de los procesos de instrucción, que además, deberían ser innovadores y capaces de adaptarse a la sociedad tecnológica actual.

La necesidad de emplear herramientas tecnológicas en la educación, lleva a los docentes a la adquisición y desarrollo de destrezas, habilidades y competencias tecnológicas, que puedan adaptarse y emplearse en el proceso educativo. Tomando en consideración, lo anterior se piensa en la necesidad de formación y desarrollo de competencias tecnológicas por parte de los docentes, para así afrontar de la mejor manera posible las nuevas circunstancias educativas del siglo XXI, en una sociedad de constantes cambios (Bosco, 2013).

Dentro de este mismo orden de ideas, diversos autores plantean que existe un interés hacia la formación y perfeccionamiento de las competencias digitales, como componente básico dentro del desarrollo profesional del docente. Es por ello que, el Instituto Nacional de Tecnologías Educativas y de Formación del Profesorado (INTEF, 2017) (España) actualizó el "Marco Común de Competencia Digital Docente", donde también se definen cinco áreas de competencia digital que se ilustran en la Figura 1. 


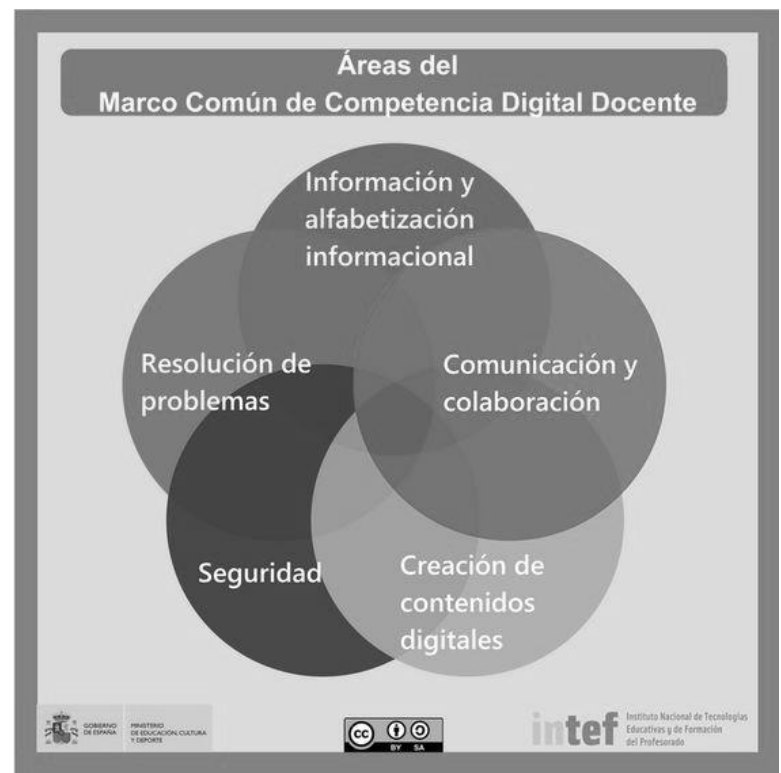

Figura 1. Áreas del Marco Común de Competencia Digital Docente. (INTEF, 2017).

Las áreas mostradas en la figura 1 se refieren a:

1. Información y alfabetización informacional: identificar, localizar, recuperar, almacenar, organizar y analizar la información digital, evaluando su finalidad y relevancia.

2. Comunicación y colaboración: comunicar en entornos digitales, compartir recursos a través de herramientas en línea, conectar y colaborar con otros a través de herramientas digitales, interactuar y participar en comunidades y redes; conciencia intercultural.

3. Creación de contenido digital: crear y editar contenidos nuevos (textos, imágenes, videos), integrar y reelaborar conocimientos y contenidos previos, realizar producciones artísticas, contenidos multimedia y programación informática, saber aplicar los derechos de propiedad intelectual y las licencias de uso.

4. Seguridad: protección personal, protección de datos, protección de la identidad digital, uso de seguridad, uso seguro y sostenible.

5. Resolución de problemas: identificar necesidades y recursos digitales, tomar decisiones a la hora de elegir la herramienta digital apropiada, acorde a la finalidad o necesidad, resolver problemas conceptuales a través de medios digitales, resolver problemas técnicos, uso creativo de la tecnología, actualizar la competencia propia y la de otros. 
Resulta claro, que la formación docente en el uso de las TIC, no solo incluye el desarrollo de habilidades y destrezas, sino de adecuada utilización de dichas herramientas en cada área de conocimiento.

La UNESCO (2008) ofreció una serie de competencias, donde se destaca como objetivo principal que las naciones elaboren políticas educativas, que favorezcan la formación de los docentes en el uso de las TIC. La idea es que las sociedades, cada vez más seguidoras de la información y su uso a través de la tecnología, tengan en cuenta lo siguiente:

- constituir fuerzas productivas dotadas de competencias en materia de TIC, que les permitan manejar la información y les proporcionen la capacidad de reflexionar, crear y solucionar problemas, a fin de generar conocimientos.

- propiciar que sus ciudadanos sean instruidos y capaces, de modo que cada uno pueda orientar con eficacia su propia vida y desarrollar una existencia plena y satisfactoria.

- alentar a todos los ciudadanos a que participen cabalmente en la sociedad e influyan en las decisiones que afectan a sus vidas.

- fomentar la comprensión intercultural y la solución pacífica de los conflictos. (sp)

De este modo las sociedades y los ciudadanos que las conforman puedan, a través de la educación, y sobre todo de la formación de sus docentes en el uso de las tecnologías, conformar verdaderas sociedades de la información y de la comunicación.

La propuesta de la UNESCO (2008) en el proyecto Estándares UNESCO de Competencias en TIC para Docentes (ECD-TIC), dentro del programa "Educación para Todos" numera un conjunto de directrices, que permiten identificar, desarrollar y evaluar materiales de aprendizaje, donde su integración al proceso de enseñanza aprendizaje, juegue un papel importante. Además, toma en cuenta la necesidad de su formación para su posterior utilización en la praxis profesional.

El proyecto ECD-TIC, posee tres enfoques básicos, que sirven para responder a los distintos objetivos y visiones en materia de políticas educativas. Se ilustran en la Figura 2. 


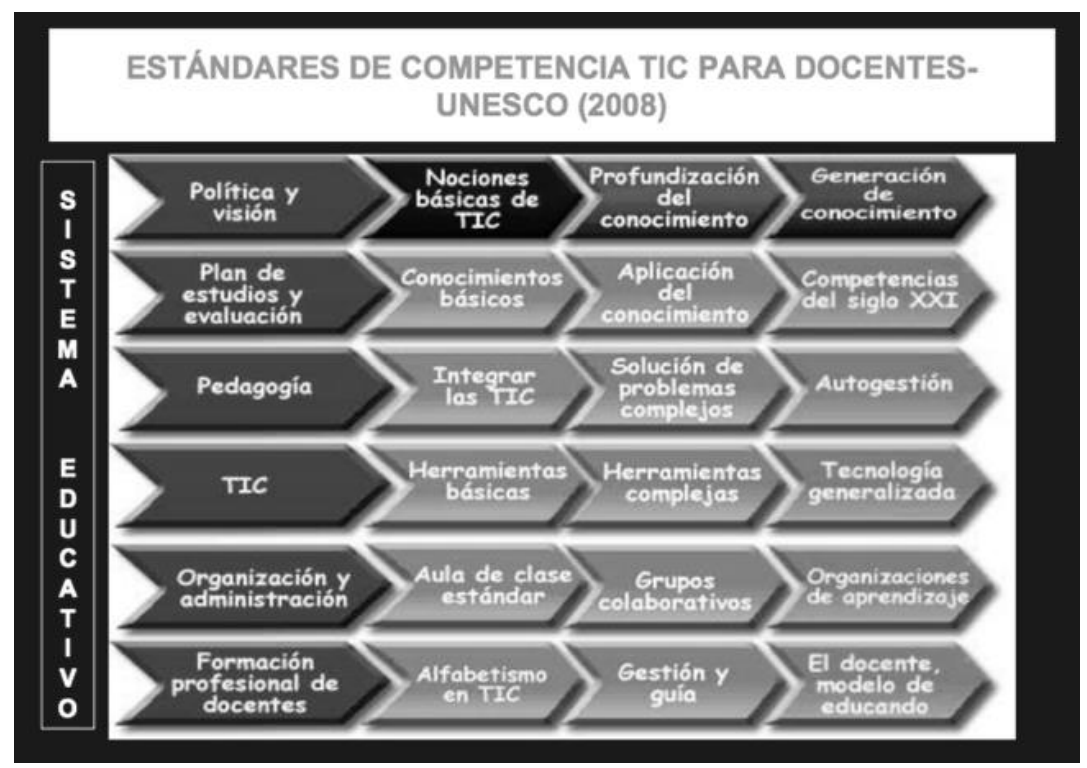

Figura 2. Estándares de Competencias TIC para Docentes. (UNESCO, 2008).

Es por ello que la UNESCO, a través de su propuesta, resume en un esquema gráfico, las competencias que se deben desarrollar en los docentes para alcanzar los estándares indicados.

Al revisar varias de las competencias, que se aprecian en la figura, pareciera evidente que algunas de ellas se podrían encontrar presente en las actividades docentes, que se realizan en los espacios de enseñanza y aprendizaje, en los cuales el uso de dichas competencias brindaría un abanico de posibilidades para hacer las clases eficaces y eficientes empleando tecnología.

\section{Marco Metodológico.}

La Universidad Central de Venezuela en conjunto con el Sistema de Actualización Docente del Profesorado (SADPRO-UCV), ofrece desde el año 2009, una formación para los nuevos profesores, que ingresan a la universidad a través de concurso, como parte de un plan de formación y capacitación, para ello ofrece el Diplomado de Formación Integral para el Docente de la UCV conocido como el Diplomado Aletheia, en el mismo ingresan anualmente un número representativo de docentes de cada facultad e instituto de investigación. 


\section{Metodología}

\section{Tipo de investigación}

Se trata de una investigación no experimental, donde se observan las variables tal como se dan en su contexto natural, para después analizarlas (Hernández et al., 2010).

Para ello se parte de una indagación acerca del uso de las competencias digitales de los profesionales que ingresan a la universidad para desarrollar praxis docente. Se ubica en un diseño que pretende investigar, en profesionales de diversas áreas del saber, su conocimiento y uso diferentes áreas tecnológicas con las competencias digitales. En tal sentido, se trata de un estudio descriptivo ya que "busca especificar propiedades, características y rasgos importantes... Describe tendencias de un grupo o población" (Hernández et al., 2010, p. 80) ubicada en una muestra de profesionales dedicados a la labor docente en la Universidad Central de Venezuela. Todo ello con la intención de verificar en este grupo el uso de las competencias digitales que puedan ser aplicadas posteriormente a la praxis profesional docente.

\section{Muestra o participantes del estudio}

La muestra estuvo conformada por 89 profesores participantes en las cohortes IX (20182019) y X (2019-2020) del Diplomado Aletheia, los integrantes de la misma pertenecen a las 11 facultades e institutos de investigación de la Universidad Central de Venezuela, distribuidos como se aprecia en la Tabla 1. La edad promedio de los participantes fue de 41 años, con un rango de edades entre los 25 y 65 años, con 50 (56\%) participantes de sexo femenino y 39 (44\%) de sexo masculino. También destaca entre los participantes, que $27(30,3 \%)$ son docentes a Dedicación Exclusiva y $26(29,2 \%)$ son tiempo completo. Asimismo, $59(66,2 \%)$ de los participantes poseen estudios de cuarto nivel (especialización y maestría). 
Tabla 1. Facultades e Institutos

\begin{tabular}{|c|c|c|c|}
\hline Facultad & Cohorte IX & Cohorte X & Porcentaje \\
\hline Agronomía & 6 & 2 & 8,9 \\
\hline Arquitectura & 0 & 2 & 2,2 \\
\hline Ciencias & 1 & 7 & 8,9 \\
\hline Ciencias Jurídicas y Políticas & 2 & 1 & 3,3 \\
\hline Ciencias Económicas y Sociales & 11 & 6 & 19,1 \\
\hline Farmacia & 2 & 3 & 5,6 \\
\hline Humanidades & 1 & 1 & 2,2 \\
\hline Ingeniería & 8 & 0 & 8,9 \\
\hline Medicina & 11 & 10 & 23,5 \\
\hline Odontología & 10 & 0 & 11,2 \\
\hline Veterinarias & 2 & 0 & 2,2 \\
\hline Otras & 3 & 0 & 3,3 \\
\hline Total & 89 & & 100 \\
\hline
\end{tabular}

Fuente: elaboración propia del autor (2020).

\section{Instrumentos y procedimientos para el análisis de los resultados}

Las técnicas e instrumentos que se emplearon para la recopilación de datos, permitieron responder a las interrogantes planteadas.

En atención a lo expuesto, se puede decir que, el proceso se enmarcó en una investigación de campo, en donde la información y los datos se recogen de la realidad; es por ello que resultó conveniente la utilización de una encuesta, como técnica para atender los objetivos de la investigación, empleando como instrumento el cuestionario como elemento para obtener la información y facilitar la economía de tiempo en la obtención de la misma, todo ello siguiendo la descripción y recomendación sugeridas por Castro (2010) y Sabino (1992).

El cuestionario utilizó la rúbrica elaborada por Álvarez (2013), como se muestra en la Figura 3, en la cual se evalúan las capacidades con cuatro niveles de consecución, donde uno corresponde al menor y cuatro al mayor logro de la competencia. La rúbrica se adaptó al instrumento diagnóstico de SADPRO-UCV, dirigido a determinar el uso de las competencias digitales para su praxis docente de los profesionales que ingresan como profesores de la Universidad Central de Venezuela, los ítems están conformados por una escala tipo Lickert, que emplea valores desde ninguna dificultad hasta mayor dificultad. La recolección de los datos se efectuó a través de un cuestionario en línea, donde el enlace se enviaba por correo electrónico a los participantes de la IX y X cohorte del Diplomado Aletheia.

Para categorizar cuantitativamente la competencia digital de los docentes, se elaboró un índice empleando el resultado de Álvarez (2013), basado en la puntuación total de cada individuo, además, se consideran los niveles de adquisición de competencia de la INTEF (2017), 
en el cual las competencias se determinan a través de tres categorías: a) básico, b) intermedio y avanzado.

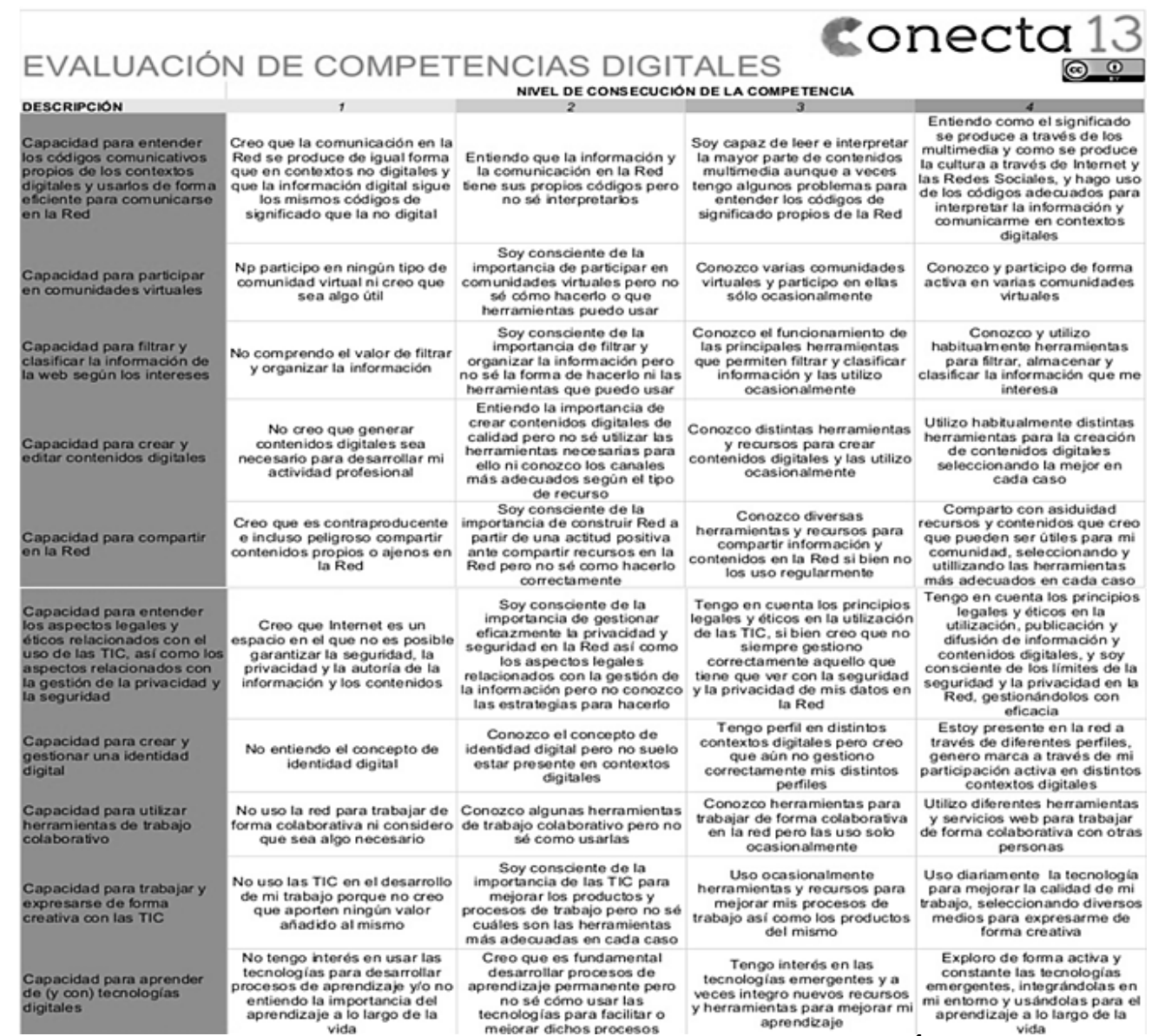

Figura 3. Rúbrica de Autoevaluación de Competencias Digitales (Álvarez, 2013)

Asimismo, las competencias digitales según el Marco Europeo (2017) implican el uso crítico y seguro de las Tecnologías de la Sociedad de la Información para el trabajo, tiempo libre y la comunicación. Apoyándose en habilidades TIC básicas: uso de computadores para recuperar, evaluar, almacenar, producir, presentar e intercambiar información, y para comunicar y participar en redes de colaboración a través de Internet.

\section{Resultados y Discusión}

Se enfatiza en el análisis e interpretación de los resultados, obtenidos una vez aplicado el instrumento a los participantes del diplomado Aletheia en su IX y X cohorte, sobre el uso de las competencias digitales para la praxis docente. 
La información se muestra a través de tablas y gráficos, en los que los datos destacan la frecuencia y porcentaje por competencia digital, con la finalidad de representar las respuestas de los profesionales docentes que forman parte de la investigación.

Para el análisis e interpretación de datos, se tomó en consideración las la frecuencia y porcentaje de las opciones seleccionadas, además, la escala planteada para enmarcar las alternativas. A continuación, se muestran los resultados.

Tabla 2 - Carga de Archivos

\begin{tabular}{cccccccc}
\hline Nivel de Dificultad & \multicolumn{2}{c}{ Cohorte IX } & \multicolumn{2}{c}{ Cohorte X } & \multicolumn{3}{c}{ Total } \\
\hline Ninguna Dificultad & $\mathrm{n}$ & $\%$ & $\mathrm{n}$ & $\%$ & $\mathrm{n}$ & $\%$ \\
\hline Dificultad muy baja & 40 & 70 & 1 & 3,1 & 41 & $\mathbf{4 6}$ \\
Ni menor ni mayor dificultad & 10 & 17,5 & 28 & 87,5 & 38 & $\mathbf{4 2 , 6}$ \\
Dificultad media & 4 & 7,0 & 1 & 3,1 & 5 & 5,6 \\
Mayor Dificultad & 1 & 1,7 & 2 & 6,2 & 3 & 3,3 \\
No sabe no responde & 1 & 1,7 & 0 & 0 & 1 & 1,12 \\
& 1 & 1,7 & 0 & 0 & 1 & 1,12 \\
\hline & & & & Total & $\mathbf{8 9}$ & $\mathbf{1 0 0}$
\end{tabular}

Fuente: elaboración propia del autor (2020).

El 88,6\% de los encuestados reportan que subir o cargar archivos en espacios virtuales o correos electrónicos les representa una dificultad muy baja o ninguna dificultad.

Tabla 3 - Participación en Foros Virtuales

\begin{tabular}{ccccccc}
\hline Nivel de Dificultad & \multicolumn{2}{c}{ Cohorte IX } & \multicolumn{2}{c}{ Cohorte X } & \multicolumn{2}{c}{ Total } \\
\hline & $\mathrm{n}$ & $\%$ & $\mathrm{n}$ & $\%$ & $\mathrm{n}$ & $\%$ \\
\hline Ninguna Dificultad & 28 & 49,1 & 15 & 46,8 & 43 & 48,3 \\
Dificultad muy baja & 14 & 24,5 & 6 & 18,7 & 20 & 22,4 \\
Ni menor ni mayor dificultad & 9 & 15,7 & 6 & 18,7 & 15 & 16,8 \\
Dificultad media & 0 & 0 & 4 & 12,5 & 4 & 4,4 \\
Mayor Dificultad & 2 & 3,5 & 1 & 3,1 & 3 & 3,3 \\
No sabe no responde & 4 & 7,0 & 0 & 0 & 4 & 4,4 \\
\hline
\end{tabular}

Fuente: elaboración propia del autor (2020).

Un 70,7\% de los participantes, expresa que participar en foros virtuales, desarrollados en plataformas digitales, les representa una dificultad muy baja o ninguna dificultad, lo que implica es una actividad donde con frecuencia se indican los pasos a seguir y las normas de funcionamiento. 
Tabla 4 - Recepción y envío de correos electrónicos

\begin{tabular}{ccccccc}
\hline Nivel de Dificultad & \multicolumn{2}{c}{ Cohorte IX } & \multicolumn{2}{c}{ Cohorte X } & \multicolumn{2}{c}{ Total } \\
\hline & $\mathrm{n}$ & $\%$ & $\mathrm{n}$ & $\%$ & $\mathrm{n}$ & $\%$ \\
\hline Ninguna Dificultad & 51 & 89,4 & 28 & 87,5 & 79 & 88,7 \\
Dificultad muy baja & 3 & 5,2 & 3 & 9,3 & 6 & 6,7 \\
Ni menor ni mayor dificultad & 0 & 0 & 1 & 3,1 & 1 & 1,1 \\
Dificultad media & 0 & 0 & 0 & 0 & 0 & 0 \\
Mayor Dificultad & 0 & 0 & 0 & 0 & 0 & 0 \\
No sabe no responde & 2 & 3,5 & 0 & 0 & 2 & 2,2 \\
\hline
\end{tabular}

Fuente: elaboración propia del autor (2020).

El 95,4\% expresa que el envío y recepción de correos electrónicos, es una actividad sin altos niveles de complejidad, que además se efectúa con mucha frecuencia en diferentes ambientes, tanto laborales como personales, siendo una herramienta de uso frecuente.

Tabla 5 - Participación en Chat

\begin{tabular}{ccccccc}
\hline Nivel de Dificultad & \multicolumn{2}{c}{ Cohorte IX } & \multicolumn{2}{c}{ Cohorte X } & \multicolumn{2}{c}{ Total } \\
\hline Ninguna Dificultad & $\mathrm{n}$ & $\%$ & $\mathrm{n}$ & $\%$ & $\mathrm{n}$ & $\%$ \\
\hline Dificultad muy baja & 38 & 66,6 & 23 & 71,8 & 61 & 68,5 \\
Ni menor ni mayor dificultad & 13 & 22,8 & 4 & 12,5 & 17 & 19,10 \\
Dificultad media & 2 & 3,5 & 3 & 9,3 & 5 & 5,6 \\
Mayor Dificultad & 0 & 0 & 1 & 3,1 & 1 & 1,1 \\
No sabe no responde & 2 & 3,5 & 1 & 3,1 & 3 & 3,3 \\
& 2 & 3,5 & 0 & 0 & 2 & 2,2 \\
\hline
\end{tabular}

Fuente: elaboración propia del autor (2020).

El 69\% señala que participar en un chat, es una actividad sin ningún nivel de dificultad, dado que muchos espacios de conversación, se realizan a través de aplicaciones que se instalan en teléfonos inteligentes, y que en los actuales momentos son de uso frecuente y cotidiano.

Tabla 6 - Carga de Imágenes

\begin{tabular}{cccccccc}
\hline Nivel de Dificultad & \multicolumn{2}{c}{ Cohorte IX } & \multicolumn{2}{c}{ Cohorte X } & \multicolumn{2}{c}{ Total } \\
\hline Ninguna Dificultad & $\mathrm{n}$ & $\%$ & $\mathrm{n}$ & $\%$ & $\mathrm{n}$ & $\%$ \\
\hline Dificultad muy baja & 38 & 66,6 & 25 & 78,1 & 63 & 70,7 \\
Ni menor ni mayor dificultad & 8 & 14,0 & 6 & 18,7 & 14 & 15,7 \\
Dificultad media & 8 & 14,0 & 1 & 3,1 & 9 & 10,1 \\
Mayor Dificultad & 0 & 0 & 0 & 0 & 0 & 0 \\
No sabe no responde & 0 & 0 & 0 & 0 & 0 & 0 \\
& 3 & 5,2 & 0 & 0 & 3 & 3,3 \\
\hline
\end{tabular}

Fuente: elaboración propia del autor (2020). 
Un $71 \%$ de la muestra manifiesta que subir o cargar imágenes en plataformas virtuales no tiene mayor nivel de dificultad y que puede ser realizado con facilidad.

Tabla 7 - Acceso a bibliografía y materiales instruccionales

\begin{tabular}{cccccccc}
\hline Nivel de Dificultad & \multicolumn{2}{c}{ Cohorte IX } & \multicolumn{2}{c}{ Cohorte X } & \multicolumn{2}{c}{ Total } \\
\hline & $\mathrm{n}$ & $\%$ & $\mathrm{n}$ & $\%$ & $\mathrm{n}$ & $\%$ \\
\hline Ninguna Dificultad & 31 & 54,3 & 19 & 59,3 & 50 & 56,1 \\
Dificultad muy baja & 19 & 33,3 & 9 & 28,1 & 28 & 31,4 \\
Ni menor ni mayor dificultad & 5 & 8,77 & 4 & 12,5 & 9 & 10,1 \\
Dificultad media & 1 & 1,7 & 0 & 0 & 1 & 1,1 \\
Mayor Dificultad & 0 & 0 & 0 & 0 & 0 & 0 \\
No sabe no responde & 1 & 1,7 & 0 & 0 & 1 & 1,1 \\
\hline
\end{tabular}

Fuente: elaboración propia del autor (2020).

Un $56 \%$ de los docentes/ de los encuetados, manifiesta que tener acceso a materiales instruccionales y bibliográficos no implica ninguna dificultad, además ya en preguntas anteriores expresaron que poseen capacidades para hacer búsqueda de información en línea, han empleado el repositorio saber UCV.

Cabe destacar que a partir de la tabla 8 se reflejan datos donde los participantes podían seleccionar varias opciones de respuesta, de acuerdo al planteamiento realizado, de allí que los resultados muestren valores diferentes al número total de participantes por cohorte.

Tabla 8 - Herramientas Tecnológicas

\begin{tabular}{ccccccc}
\hline & \multicolumn{2}{c}{ Cohorte IX } & \multicolumn{2}{c}{ Cohorte X } & \multicolumn{2}{c}{ Total } \\
\hline & $\mathrm{n}$ & $\%$ & $\mathrm{n}$ & $\%$ & $\mathrm{n}$ & $\%$ \\
\hline Curso en línea & 22 & 38,5 & 19 & 59,3 & 41 & 46,0 \\
Foro Virtual & 24 & 42,1 & 17 & 53,1 & 41 & 46,0 \\
Wikipedia & 25 & 43,8 & 15 & 46,8 & 40 & 44,9 \\
Blogs & 25 & 43,8 & 18 & 56,2 & 43 & 48,3 \\
Grupos Web & 11 & 19,2 & 12 & 37,5 & 23 & 25,8 \\
Correos electrónicos & 53 & 92,9 & 32 & 100 & 85 & 95,5 \\
Webex & 0 & 0 & 0 & 0 & 0 & 0 \\
Blacboard & 4 & 7,0 & 0 & 0 & 4 & 4,4 \\
Flash 5 & 4 & 7,0 & 0 & 0 & 4 & 4,4 \\
Excel & 41 & 71,9 & 31 & 96,8 & 72 & 80,8 \\
Publisher & 14 & 24,5 & 15 & 46,8 & 29 & 32,5 \\
Power Point & 51 & 89,4 & 32 & 100 & 83 & 93,2 \\
Facebook & 28 & 49,1 & 14 & 43,7 & 42 & 47,1 \\
Twitter & 25 & 43,8 & 14 & 43,7 & 39 & 43,8 \\
Moodle & 9 & 15,7 & 7 & 21,8 & 16 & 17,9 \\
Otro & 10 & 17,5 & 7 & 21,8 & 17 & 19,1 \\
\hline
\end{tabular}




\section{Erika Machado}

Fuente: elaboración propia del autor (2020).

Al hacer mención a las herramientas tecnológicas usadas durante la actividad académica de los participantes, destacan de los resultados y del análisis que:

Un 96\% de la muestra posee destrezas y capacidades para la utilización de correos electrónicos, un medio de comunicación de uso frecuente y cotidiano en todos los ámbitos, sociales, académicos, laborales, entre otros. Así como la habilidad, para utilizar el procesador de texto en diversas actividades profesionales y docentes.

El $81 \%$ expresa que emplea o ha empleado, la hoja de cálculo para la realización de actividades contables y de ayuda a los procesos de control de calificaciones, propios de la labor docente.

Un 93\% indica que emplea o ha empleado, las presentaciones electrónicas, como un software que permite generar materiales de apoyo, para ser empleados en el aula de clases.

También destaca que un $46 \%$ ha utilizado cursos en línea, foros virtuales, blogs y Wikipedia como herramientas de aprendizaje, en las que una persona puede tener tanto el rol de estudiante como de facilitador, además, es importante señalar que 18\% (17 docentes) de los encuestados han utilizado el campus virtual de la universidad con fines educativos y de apoyo a su labor académica y profesional.

Asimismo, los datos pueden reafirmar lo señalado por la UNESCO (2008), según la cual “gracias a la utilización continua y eficaz de las TIC en procesos educativos, los estudiantes tienen la oportunidad de adquirir capacidades en el uso de éstas". Por lo cual los resultados anteriores, reflejan que emplear diversas herramientas tecnológicas puede tener una incidencia en el proceso educativo y en el aprendizaje de los estudiantes.

Tabla 9 -Tratamiento de la Información

\begin{tabular}{|c|c|c|c|c|c|c|}
\hline & \multicolumn{2}{|c|}{ Cohorte IX } & \multicolumn{2}{|c|}{ Cohorte X } & \multicolumn{2}{|c|}{ Total } \\
\hline & $\mathrm{n}$ & $\%$ & $\mathrm{n}$ & $\%$ & $\mathrm{n}$ & $\%$ \\
\hline $\begin{array}{l}\text { Clasifico la información de una manera metódica usando archivos y } \\
\text { carpetas }\end{array}$ & 16 & 28,0 & 31 & 96,8 & 47 & 52,8 \\
\hline $\begin{array}{l}\text { Comparo diferentes fuentes para evaluar la fiabilidad de la información } \\
\text { que encuentro. }\end{array}$ & 4 & 7,0 & 28 & 87,5 & 32 & 35,9 \\
\hline Puedo buscar información online utilizando un motor de búsqueda. & 12 & 21,0 & 31 & 96,8 & 43 & 48,3 \\
\hline Puedo usar estrategias avanzadas de búsqueda & 3 & 5,2 & 15 & 46,8 & 18 & 20,2 \\
\hline $\begin{array}{l}\text { Sé evaluar la validez y la credibilidad de la información usando } \\
\text { variedad de criterios. }\end{array}$ & 7 & 12,2 & 23 & 71,8 & 30 & 33,7 \\
\hline Sé guardar información encontrada en internet en diferentes formatos & 11 & 19,2 & 26 & 81,2 & 37 & 41,5 \\
\hline Soy capaz de guardar y almacenar archivos o contenido & 2 & 3,5 & 31 & 96,8 & 33 & 37,0 \\
\hline Se utilizar diferentes motores de búsqueda para encontrar información. & 0 & 0 & 29 & 90,6 & 29 & 32,5 \\
\hline
\end{tabular}


Fuente: elaboración propia del autor (2020).

El 53\% de los encuestados/docentes/profesores/de la muestra tiene la capacidad de buscar, guardar y clasificar información en archivos y carpetas, es decir, posee habilidades para efectuar búsqueda e internet. Asimismo se pudo evidenciar que/conocer que un $48 \%$ tiene habilidades para buscar información empleando motores de búsqueda dificultades, sin embargo, $20 \%$ presenta dificultades para usar estrategias avanzadas de búsqueda de información, lo cual indica, la necesidad de ayudarles para mejorar las competencias y habilidades en cuanto a las estrategias más eficaces y eficientes para efectuar búsquedas en internet a objeto de que desarrollen las destrezas para tal fin. Es importante destacar que los resultados anteriores, muestran como la tecnología enmarcada por la globalización del conocimiento y la sociedad de la información, pueden brindar beneficios en la labor docente, es por ello que, Pérez Zúñiga, Mercado Lozano, Martínez García y Mena Hernández (2018) enfatizan acerca de:

La Sociedad de información y la Sociedad del conocimiento son dos expresiones que en el campo educativo se refieren al uso de dispositivos digitales para facilitar el aprendizaje y consolidar un modelo integral de educación que cumpla con los objetivos tecno-pedagógicos de la actualidad.

Por lo cual, el uso y conocimiento de herramientas tecnológicas por parte de los docentes llega a tener una incidencia en su praxis profesional en la educación universitaria.

Tabla 10 - Creación de Contenidos

\begin{tabular}{lrrrrrrr}
\hline & Cohorte IX & \multicolumn{2}{c}{ Cohorte X } & \multicolumn{2}{c}{ Total } \\
\hline & $\mathrm{n}$ & $\%$ & $\mathrm{n}$ & $\%$ & $\mathrm{n}$ & $\%$ \\
\hline Puedo producir contenido digital simple & 18 & 31,5 & 32 & 100 & 50 & 56,1 \\
Puedo editar el contenido producido por otros. & 2 & 3,5 & 17 & 53,1 & 19 & 21,3 \\
Puedo aplicar y modificar la configuración del software & 2 & 3,5 & 12 & 37,5 & 14 & 15,7 \\
Soy capaz de crear contenidos digitales complejos. & 14 & 24,5 & 11 & 34,3 & 23 & 25,8 \\
Sé aplicar formatos básicos & 12 & 21,0 & 29 & 90,6 & 41 & 46,0 \\
Sé cómo hacer referencias y reusar el contenido protegido & 3 & 5,2 & 7 & 21,8 & 10 & 11,2 \\
Puedo producir o modificar contenido multimedia. & 1 & 1,1 & 7 & 21,8 & 8 & 8,9 \\
Puedo crear una página web & 0 & 0 & 6 & 18,7 & 6 & 6,7 \\
Sé cómo aplicar licencias y copyright. & 0 & 0 & 1 & 3,1 & 1 & 1,1 \\
Puedo usar varios lenguajes de programación. & 2 & 2,2 & 0 & 0 & 2 & 2,2 \\
\hline
\end{tabular}

Fuente: elaboración propia del autor (2020).

Un 56\% tiene capacidades y destrezas para generar contenidos digitales empleando formatos o software básicos para ello, es decir, puede crear infografias, mapas mentales o conceptuales, presentaciones electrónicas, elementos de uso común en los procesos de enseñanza y aprendizaje, sin embargo, los resultados indican que en otras destrezas se podrían 
establecer procesos de capacitación para aumentar el uso de herrmaientas para diseñar y crear elementos de más nivel y trabajo, por ejemplo podcast, vídeos, wikis, animaciones, entre otras.

Tabla 11 - Comunicación en línea

\begin{tabular}{lccccccc}
\hline & \multicolumn{2}{c}{ Cohorte IX } & \multicolumn{2}{c}{ Cohorte X } & \multicolumn{3}{c}{ Total } \\
\hline & $\mathrm{n}$ & $\%$ & $\mathrm{n}$ & $\%$ & $\mathrm{n}$ & $\%$ \\
\hline Puedo comunicar utilizando el teléfono móvil & 15 & 26,3 & 32 & 100 & 47 & 52,8 \\
Puedo usar tecnología digital para interactuar & 1 & 1,7 & 30 & 93,7 & 31 & 34,8 \\
Conozco la existencia de redes sociales e instrumentos de & 8 & 14,0 & 32 & 100 & 40 & 44,9 \\
colaboración. & 1 & 1,7 & 26 & 81,2 & 27 & 30,3 \\
Puedo usar características avanzadas de varias aplicaciones & 1 & 1,7 & 26 & 81,2 & 27 & 30,3 \\
Puedo usar herramientas de colaboración & 4 & 7,0 & 28 & 87,5 & 32 & 35,9 \\
Transmito y comparto conocimientos con otros en línea. & 0 & 0 & 13 & 40,6 & 13 & 14,6 \\
Soy consciente del uso de las normas para comunicación en línea & 15 & 26,3 & 30 & 93,7 & 45 & 50,5 \\
Uso activamente variedad de aplicaciones de comunicación & 2 & 3,5 & 12 & 37,5 & 14 & 15,7 \\
Creo y gestiono contenidos con herramientas de colaboración & 8 & 14,0 & 21 & 65,6 & 29 & 32,5 \\
Participó activamente en espacios online & & & &
\end{tabular}

Fuente: elaboración propia del autor (2020).

El $53 \%$ posee destrezas en el uso de teléfonos moviles y de aplicaciones empleadas para los procesos de comunicación, es decir, emplean correo electrónico, servicio de mensajería (msm) y algunas redes sociales como Whatsapp, algunas de ellas de uso obligatorio para diferentes actividades que se efectuan en el mundo académico y laboral, asimismo se pudo conocer que requieren formación para desarrollar competencias para la creación y gestión de contenidos con herramientas avanzadas.

Asimismo Barnett (2009) consideraba que la universidad se va transformando en función del momento histórico que vive, entendiendo el papel del conocimiento que desea promover, generar y difundir. De allí pues, la importancia de que los docentes usen de forma eficaz y eficiente, las herramientas de comunicación existentes para su praxis profesional. 


\section{Tabla 12 - Seguridad}

\begin{tabular}{lcccccc}
\hline & \multicolumn{2}{c}{ Cohorte IX } & \multicolumn{2}{c}{ Cohorte X } & \multicolumn{2}{c}{ Total } \\
\hline & $\mathrm{n}$ & $\%$ & $\mathrm{n}$ & $\%$ & $\mathrm{n}$ & $\%$ \\
\hline Toma medidas básicas para proteger dispositivos & 17 & 29,8 & 29 & 90,6 & 46 & 51,6 \\
Es consciente que sus datos pueden ser robados & 11 & 19,2 & 32 & 100 & 43 & 48,3 \\
Sabe que no debe revelar información privada en la red & 3 & 5,2 & 32 & 100 & 35 & 39,0 \\
Toma medidas básicas para ahorrar energías & 0 & 0 & 0 & 0 & 0 & 0 \\
Ha instalado software de seguridad para dispositivos electrónicos & 5 & 8,7 & 27 & 84,3 & 32 & 35,9 \\
Utiliza diferentes contraseñas para acceder a servicios digitales & 6 & 10,5 & 30 & 93,7 & 38 & 42,6 \\
Identifica páginas o correos que pueden ser dañinos o & 0 & 0 & 19 & 59,3 & 19 & 21,3 \\
fraudulentos. & 0 & 0 & 4 & 12,5 & 4 & 4,4 \\
Configura identidad digital y sigue huella digital & 1 & 1,7 & 25 & 78,1 & 26 & 29,2 \\
Entiende de riesgos laborales asociados a la tecnología & 2 & 3,5 & 11 & 34,3 & 13 & 14,6 \\
Revisa configuración de seguridad de los sistemas & 0 & 0 & 19 & 59,3 & 19 & 21,3 \\
Sabe cómo reaccionar ante un virus informático & 5 & 8,7 & 14 & 43,7 & 19 & 21,3 \\
Configura y modifica el cortafuegos de los dispositivos & 0 & 0 & 11 & 34,3 & 11 & 12,3 \\
electrónicos & 2 & 3,5 & 20 & 62,5 & 22 & 24,7 \\
Sabe encriptar archivos y correos & 3 & 5,2 & 21 & 65,6 & 24 & 26,9 \\
Usa razonablemente la tecnología para evitar problemas de salud & & & & &
\end{tabular}

Fuente: elaboración propia del autor (2020).

Al hacer referencia a los aspectos vinculados a la seguridad tecnológica, el $52 \%$ de indicó que emplea medidas básicas para proteger sus dispositivos, tales como celulares, laptos y computadoras de escritorio, entre las medidas fundamentales se encuentran el uso de contraseñas para el acceso, uso de cargadores, uso de antivirus, protectores de pantalla, limpieza de pantallas.

Un elemento a destacar es la necesidad de los participantes por conocer, crear y configurar una identidad digital, así como las implicaciones que ésta posee en su labor docente.

Tabla 13 - Resolución de Problemas tecnológicos 


\begin{tabular}{lcccccc}
\hline & & & & & \\
& Cohorte IX & \multicolumn{2}{c}{ Cohorte X } & \multicolumn{2}{c}{ Total } \\
\hline & $\mathrm{n}$ & $\%$ & $\mathrm{n}$ & $\%$ & $\mathrm{n}$ & $\%$ \\
\hline Es capaz de encontrar apoyo y asistencia en un problema técnico & 6 & 10,5 & 26 & 68,7 & 32 & 35,9 \\
Sabe cómo solucionar problemas rutinarios & 10 & 17,5 & 32 & 100 & 42 & 47,1 \\
Sabe que los útiles digitales ayudan a resolver problemas & 3 & 5,2 & 0 & 0 & 3 & 3,3 \\
Es consciente de sus limitaciones para resolver problemas & 2 & 3,5 & 22 & 68,7 & 24 & 26,9 \\
Es consciente que necesita actualizar sus habilidades digitales & 13 & 22,8 & 32 & 100 & 45 & 50,5 \\
Puede resolver con frecuencia los problemas que surgen de usar & 4 & 7,0 & 19 & 59,3 & 23 & 25,8 \\
tecnología & & 0 & 0 & 0 & 0 & 0 \\
Puede seleccionar un instrumento que se adapte a sus necesidades & 0 & 0 & & & \\
Puede resolver problemas tecnológicos revisando las opciones de los & 3 & 5,2 & 16 & 50 & 19 & 21,3 \\
programas & 1 & 1,7 & 20 & 62,5 & 21 & 23,5 \\
Regularmente actualiza las competencias tecnológicas & 6 & 10,5 & 10 & 31,2 & 16 & 17,9 \\
Puede resolver casi todos los problemas por uso de la tecnología & 3 & 5,2 & 17 & 53,1 & 20 & 22,4 \\
Es consciente de los avances tecnológicos & 4 & 7,0 & 12 & 37,5 & 16 & 17,9 \\
\hline Frecuentemente actualiza sus competencias tecnológicas & & & & & & \\
\hline
\end{tabular}

Fuente: elaboración propia del autor (2020).

En cuanto a la resolución de problemas tecnológicos, destaca el hecho de que un 51\% de los participantes reconoce que necesitan mantenerse actualizados en todo lo relacionado con la tecnología y educación, y sobre todo en encontrar mecanismos para solventar situaciones vinculadas a las actividades tecnológicas y los dispositivos vinculados con ello, sin embargo, un $47 \%$ expresa que posee capacidades para solucionar problemas que se le presentan cotidianamente, no necesariamente vinculados con la tecnología. 


\section{Conclusiones}

En consideración a los objetivos de estudio y al análisis e interpretación de los resultados, se llegó a las siguientes conclusiones:

En cuanto, a lo referido al uso de las competencias digitales para la praxis docentes, los participantes se encuentran en niveles bajos, según el modelo de competencias de la INTEF (2017), donde se referencias cinco áreas tecnológicas, en las cuales el profesional de la docencia requiere desarrollar destrezas y habilidades tecnológicas que pueda aplicar sus funciones académicas de formación con estudiantes.

Por consiguiente, según el modelo INTEF (2017), las áreas de competencias obtuvieron los siguientes resultados: Información y Alfabetización Informacional (60\%), Comunicación y colaboración (59\%), Creación de Contenidos Digitales (21,5\%), Seguridad (34\%), Resolución de problemas (20\%), dichos porcentajes expresan en lo referidos a Información y alfabetización Informacional, Comunicación y Colaboración que las destrezas y habilidades se encuentran en un nivel intermedio, mientrás que las restantes áreas expresan un nivel básico.

Por otra parte, el conocer dichos resultados permite establecer estrategias pedagógicas, en las cuales las competencias digitales sean un elemento transversal dentro del Diplomado Aletheia, asimismo, el desarrollo de habilidades y destrezas digitales forme parte de un plan de formación permanente, por lo cual se propone la posibilidad de establecer politicas universitarias en las que la formación en competencias digitales, sea parte de la formación de todos los profesionales que ingresan como docentes a la universidad.

Asimismo, el dominio de las competencias digitales en los docentes universitarios, podría convertirse en un abanico de posibilidades, para incrementar la eficacia y eficiancia de las clases.

\section{Referencias}

Acaso, M. (2013). rEDUvolution Hacer la Revolución en la Educación. CIUDAD: Paidós S.A.

Álvarez, D. (2013). Rúbrica de evaluación de competencias digitales. Disponible en http://bit.ly/2yFllHs

Barnett, R. (2009). Universidades, conocimiento y planes de estudio. Conferencia presentada en el III Seminario Internacional: Universidad y Sociedad del Conocimiento. Desafíos y Tendencias. Junio. Red UNISIC. Universidad Santiago de Compostela. 
Badilla, M. (2016). Análisis y evaluación de un modelo socioconstructivo de formación permanente del profesorado para la incorporación de las TIC. [Documento en línea] Disponible en: https://text-mx.123dok.com/document/7qvx95gy-analisis-y-evaluacionde-un-modelo-socioconstructivo-de-formacion-permanente-del-profesorado-para-laincorporacion-de-las-tecnologias-de-informacion-y-comunicacion-estudio-del-casocetei-del-proceso-de-integracion-pedagogica-de-la-pizarra-digital.html

Bosco, A. (2013). Las Tecnologías de la Información y la Comunicación en la Formación del Profesorado: Lineamientos, Actualidad y Prospectiva. RAZÓN y PALABRA. Revista Digital en Iberoamérica Especializada en Comunicología. Disponible en http://www.razonypalabra.org.mx/n63/abosco.html

Castro, G. (2010). Fuentes y técnicas de recolección de información. [Documento en línea] Disponible en http://es.slideshare.net/Giovannycastromz/fuentes-y-tecnicas-de-recoleccion-deinformacion

Cañuta, J. (s/f). Actitud de los Docentes frente al uso de Nuevas Tecnologías. Universidad de Playa Ancha. Revisado en: http://www.slideshare.net/ jcanuta/tesis-actitud-de-losdocentes-frente-al-uso-de-las-tics.

Fernández, R. (2010). Nuevas Tecnologías y Formación del Profesorado. [Documento en línea] Disponible en http://es.slideshare.net/Ricardo.FM/nuevas-tecnologas-y-formacindel-profesorado

Flores, S. (s/f). Competencias docentes para el uso de las TICs. Revisado en: http://www.slideshare.net/tchcarrasco/competencias-docentes-para-el-uso-de-las-tic$\underline{3850187}$

Hernández R., Fernández, C, Baptista, P. (2010). Metodología de la investigación. México: McGraw Hill.

INTEF Instituto Nacional de Tecnologías Educativas y de Formación del Profesorado (2017). Marco Común de Competencia Digital Docente. [en línea] Disponible en: http://educalab.es/documents/10180/12809/Marco+competencia+digital+docente+2017/afb079 87-1ad6-4b2d-bdc8-58e9faeeccea

Larrea, C. (2013) Integración curricular de las TIC en los docentes de Lengua y Literatura en los niveles de Educación Básica y Bachillerato en las Unidades Educativas Experimentales "Eugenio Espejo", "Sebastián de Benalcázar" y "Celestin Freiner", en el año lectivo 2012-2013. [Documento en línea] Disponible en http://dspace.utpl.edu.ec/handle/123456789/7541?locale=es

López, E. (2011). La Cultura Tecnológica: Implicaciones en la Formación Docente. [Documento en línea] Disponible en http://es.slideshare.net/EDLG/edgar-daniellopezlaculturatecnolgica

Lorenzo, G., Pomares, J. y Roib, R.(s/f). Diseño de un modelo de indicadores de competencias en la TIC en la Docencia Universitaria. Universidad de Alicante. España. [Documento en línea] Disponible en http://www.upm.es/ sfs/E.T.S.I.\%20Montes/Sub.\%20Calidad/Recursos\%20Competencias/Archivos/Univ_ Alicante_Indicadores.pdf 
Martínez, R., Leite, C. y Monteiro, A. (2000). TIC y formación inicial de maestros: oportunidades y problemas desde la perspectiva de estudiantes. [Documento en línea] Disponible en https://revistas.ort.edu.uy/cuadernos-de-investigacion-educativa/ article/view/2577

Paz, Y. (2017) Programa Instruccional de Alfabetización Tecnológica dirigido a los Docentes del Nivel de Educación Media General. [Documento en línea] Disponible en http://saber.ucv.ve/handle/123456789/17437

Pere Marqués, G. (s/f). Los Docentes: Funciones, Roles, Competencias Necesarias, Formación. [Documento en línea] Disponible en:http://peremarques.pangea.org/ docentes.htm

Pere Marqués, G. (2007). Factores de Buena Práctica Educativa con Apoyo en TIC. Trabajo de grado de maestría no publicado. Barcelona. España. [Documento en línea] Disponible: http://www.raco.cat/index.php/Educar/article/view File/76748/99171

Pere Marqués, G. (2000). Las TIC y sus Aportaciones a la Sociedad. [Documento en línea] Disponible en:https://docs.google.com/document/d/1rKWgUcP2MkU frYAQm1j6pWeuSfan3xCPvEUt4vfxQJE/edit?hl=es

Pérez Zúñiga. R, Mercado Lozano, P., Martínez García, M. y Mena Hernández, E. (2018) La Sociedad del conocimiento y la sociedad de la información como la piedra angular en la innovación tecnológica educativa. RIDE, Vol. 8, Núm. 16. Disponible en http://www.scielo.org.mx/pdf/ride/v8n16/2007-7467-ride-8-16-00847.pdf

Prendes, M. (2001). Las Nuevas Tecnologías en la Formación del Profesorado. [Documento en línea] Disponible en http://www.educarm.es/templates/portal/ images/ficheros/revistaEducarm/4/08.pdf

Programa de las Naciones Unidas para el Desarrollo. http://www.ve.undp.org/ content/venezuela/es/home/post-2015/sdg-overview/goal-9.html

Ramos, M. (2015). ¿Qué es la práctica educativa? Educ@ @nos. Revista Educativa. Disponible en http://revistaeducarnos.com/2015/12/16/que-es-la-practica-educativa/

Sabino, C. (1992). El proceso de Investigación. Libro en línea. Disponible en https://ecaths1.s3.amazonaws.com/metodologiadelainvestigacion/41237355.Sabino\% 2.

UNESCO (2008). Marco de competencias de los docentes en materia de TIC de la UNESCO. Disponible en: http://www.unesco.org/new/es/unesco/themes/icts /teachereducation/unesco-ict-competency-framework-for-teachers/

UNESCO (2008). Estándares de Competencias en TIC para Docentes. [Documento en línea] Disponible en http://www.oei.es/tic/UNESCO EstandaresDocentes.pdf

UNESCO (2015). Las TIC en la Educación. [Documento en línea] Disponible en http://www.unesco.org/new/es/unesco/themes/icts/ 
Erika Machado Infante

Profesor Mención Informática.

Universidad Pedagógica Experimental Libertador - Instituto Pedagógico de Caracas (2004) Magister Scentiarium en Educación mención Tecnologías de la Información y Comunicación. Universidad Central de Venezuela (2017). Doctorante en Educación. Línea de Investigación Tecnologías de la Información y Comunicación. Universidad Católica Ándres Bello. Adscripción:

Sistema de Actualización Docente del Profesorado de la Universidad Central de Venezuela (SADPRO-UCV). Líneas de Investigación: Educación virtual, Tecnologías de la Información y la Comunicación (TIC), profesoraerika@gmail.com

Código ORCID: http://orcid.org/0000-0002-5277-6543

\section{Como citar el artículo:}

Machado, E. Competencias Digitales de profesionales que inician su carrera universitaria como docentes. Revista Paradigma Vol. XLII, Nro. 2, Diciembre de 2021 / 256 - 278. DOI: https://doi.org/10.37618/PARADIGMA.1011-2251.2021.p256-278.id954 